\title{
АДАПТАЦИЯ ТЕХНОЛОГИИ ИНТЕРФЕЙСОВ МОЗГ-КОМПЬЮТЕР НА ВОЛНЕ РЗОО ДЛЯ ОЦЕНИВАНИЯ СОСТОЯНИЯ БОЛЬНЫХ НЕРВНОЙ АНОРЕКСИЕЙ
}

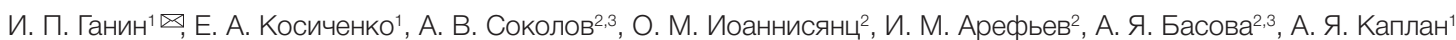

${ }^{1}$ Биологический факультет, Московский государственный университет имени М. В. Ломоносова, Москва, Россия

2 Научно-практический центр психического здоровья детей и подростков имени. Г. Е. Сухаревой, Москва, Россия

${ }^{3}$ Российский национальный исследовательский медицинский университет имени Н. И. Пирогова, Москва, Россия

Интерфейс мозг-компьютер на основе волны РЗ00 (ИМК-РЗ00) позволяет с помощью показателей электроэнцефалограммы (ЭЭГ), полученных при реакции на заданный заранее стимул, активировать соответствующую целевую команду. С помощью аналогичного алгоритма можно выделить из окружающего контекста субъективно выделяющийся по эмоциональным характеристикам стимул даже без привлечения к нему активного внимания. Такие системы позволят оценивать значимость для человека определенной информации, что можно использовать при диагностике нарушений эмоционального восприятия или системы ценностей, например, при нарушениях пищевого поведения. Целью исследования было изучить ЭЭГ-реакции на предъявление стимулов различной эмоциональной значимости больным с диагнозом F50.0 «Нервная анорексия» ( $n=12$, возраст 11-16 лет) и проверить гипотезу о возможном детектировании фокуса внимания к субъективно значимым стимулам на основе ИМК-РЗоО. Критерии включения пациентов в исследование: наличие диагноза F50.0 «Нервная анорексия»; период реабилитации. Регистрировали ЭЭГ на фроне предъявления изображений различного содержания. Выделяли и анализировали потенциалы, связанные с событиями (ПСС) с помощью cреды MATLAB 7.1 (MathWorks; CШA). Статистический анализ данных выполняли с помощью пакета программ STATISTICA 7.0 (StatSoft; CША). Как показало исследование, предъявление в условиях пассивного внимания изображений частей тела истощенных людей на фоне прочих изображений вызывало более высокие амплитуды ПСС, чем при предъявлении изображений пищи. Алгоритм позволил также распознавать реакции внимания в ЭЭГ пользователя на оба типа изображений среди остальных стимулов, при этом точность распознавания для изображений частей тела была существенно выше (89\% против 59\%). Это доказывает возможность использования ИМК-РЗО0 для распознавания неявных эмоциональных фокусов внимания и дополняет существующие знания о механизмах развития нервной анорексии.

Ключевые слова: интерфейс мозг-компьютер (ИМК), электроэнцефалограмма (ЭЭГ), потенциалы, связанные с событиями (ПСС), зрительное внимание, волна Р300, нервная анорексия, нарушение пищевого поведения, расстройство приема пищи

Информация о вкладе авторов: все авторы участвовали в планировании эксперимента; И. П. Ганин - проведение исследования, анализ и интерпретация данных, анализ литературы, подготовка текста рукописи; Е. А. Косиченко - проведение исследования, анализ литературы, анализ данных; А. В. Соколов - проведение исследования, интерпретация данных, редактирование текста; О. М. Иоаннисянц - диагностика и подбор пациентов для исследования; И. М. Арефьев - обеспечение экспериментов, интерпретации данных; А. Я. Басова - интерпретация данных, редактирование текста; А. Я. Каплан - интерпретация данных.

Соблюдение этических стандартов: исследование одобрено этическим комитетом ГБУЗ «НПЦ ПЗДМ им. Г. Е. Сухаревой ДЗМ» (протокол № 1 от 21 сентября 2017 г.). Законные представители детей подписали добровольное информированное согласие на участие в исследовании.

$\bowtie$ Для корреспонденции: Илья Петрович Ганин Ленинские горы, д. 1, стр. 12, к. 246, г. Москва,119234; ipganin@mail.ru

Статья получена: 08.10.2018 Статья принята к печати: 27.03.2019 Опубликована онлайн: 10.04.2019

DOI: $10.24075 /$ vrgmu.2019.022

\section{ADAPTING THE P300 BRAIN-COMPUTER INTERFACE TECHNOLOGY TO ASSESS CONDITION OF ANOREXIA NERVOSA PATIENTS}

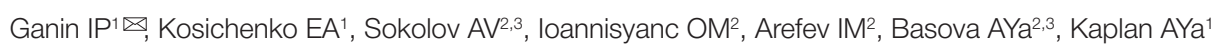

${ }^{1}$ Faculty of Biology, Lomonosov Moscow State University, Moscow, Russia

2 Scientific-practical Children's and Adolescents Mental Health Center n.a. G. Sukhareva, Moscow, Russia

${ }^{3}$ Pirogov Russian National Research Medical University, Moscow, Russia

Brain-computer interface based on the P300 wave (P300 BCl) allows activating a given command according to the electroencephalogram (EEG) response to a predetermined relevant stimulus. The same algorithm enables detecting a subjectively important item (i.e., one triggering emotional response) in an environment even without actively drawing attention to it. Such systems allow assessing the personal significance of certain information, which can be used in the diagnostics of disorders of emotional perception or value system, e.g., eating disorders. This study aimed to investigate the EEG responses of anorexia nervosa patients (diagnosis F50.0, $n=12$, age 11-16 years) to the stimuli with different perceived emotional significance, as well as to validate application of P300 BCl to detect the focus of attention to subjectively important stimuli. The inclusion criteria were: diagnosed anorexia nervosa (diagnosis F50.0); active rehabilitation. We registered the EEG while presenting images with different content to the patients. The event-related potentials (ERP) were detected and analyzed with the help of MATLAB 7.1 (MathWorks; USA). Statistica 7.0 software (StatSoft; USA) was used for statistical analysis of the data. We have discovered that in passive viewing paradigm, images of body parts of emaciated people among other images caused ERP with higher amplitude than images of food. Moreover, the accuracy of detection was higher for images of body parts: $89 \%$ against 59\%, respectively. Thus, we have proven the validity of applying P300 BCl to detect covert emotional foci of attention and added to the existing knowledge about the mechanisms of development of anorexia nervosa.

Keywords: brain-computer interface (BCl), electroencephalogram (EEG), event-related potentials (ERP), visual attention, P300 wave, eating disorders, anorexia nervosa

Author contribution: all authors participated in the experiment planning; Ganin IP — immediate research activities, data analysis and interpretation, literature analysis, manuscript authoring; Kosichenko EA — immediate research activities, literature analysis, data analysis; Sokolov AV — immediate research activities, data interpretation, text editing; loannisyanc OM — diagnosing and selection of patients for the study; Arefev IM — support of experiments, data interpretation; Basova AYa — data interpretation, text editing; Kaplan AYa — data interpretation.

Compliance with ethical standards: the study was approved by the ethical committee of Scientific-practical Children's and Adolescents Mental Health Center n.a. G. Sukhareva (Protocol №. 1 of 2017.09.21). The legal representatives of children signed a voluntary informed consent allowing them to participate in the study.

$\triangle$ Correspondence should be addressed: llya P. Ganin

Leninskie Gory 1, bld. 12, ap. 246, Moscow, 119234; ipganin@mail.ru

Received: 08.10.2018 Accepted: 27.03.2019 Published online: 10.04.2019

DOI: 10.24075/brsmu.2019.022 
В настоящее время технология интерфейсов мозг-компьютер (ИМК) входит в стандартный исследовательский инструментарий все большего числа нейрофизиологических лабораторий мира [1]. ИМК является научно-инженерной разработкой, нацеленной на обеспечение людей с утраченными или поврежденными двигательными функциями возможностью передачи команд к внешним исполнительным устройствам за счет декодирования специфических паттернов электроэнцефалограммы (ЭЭГ) [2]. Развитие исследований ИМК определяется их острой востребованностью в медицине, в частности, для управления вспомогательными устройствами [3, 4], в реабилитационных целях [5], а также для коммуникации [6].

Нынешний уровень развития технологии ИМК позволяет в некоторых случаях на основе анализа сигнала ЭЭГ распознавать команды оператора ИМК с довольно высокой точностью, что говорит о возможном расширении потенциала использования данной технологии. В первую очередь подходы и алгоритмы, используемые в ИМК, можно применять не только для распознавания конкретных команд, ассоциированных с произвольной концентрацией внимания пользователя, но и для детектирования неявных фокусов внимания к внешним стимулам или собственным внутренним состояниям. Такое непроизвольное смещение интереса или, напротив, отсутствие в ЭЭГ признаков наличия внимания в определенных условиях, в частности может быть использовано в системах инструментальной диагностики различных нарушений психики.

Среди возможных вариантов ИМК для разработки подобных систем наиболее подходящим является ИМК на основе волны Р300, или ИМК-Р300 [2, 6, 7]. В данном интерфейсе выбор команд основан на предъявлении человеку внешних стимулов-команд. Последовательно активируя стимулы (например, подсвечивая буквь на экране компьютера) и анализируя ЭЭГ-ответы на разные стимулы, система тем самым может определить целевую команду. Она детектируется по увеличенной амплитуде потенциалов, связанных с событиями (ПСС) в ответ на целевой, или значимый, для пользователя стимул. Основным компонентом ПСС является хорошо известная волна Р300, имеющая наибольшую амплитуду именно в реакциях на редкие и значимые стимулы, поэтому данный тип ИМК и получил упомянутое выше название.

Для возникновения в ЭЭГ специфичного ответа на внешний стимул требуется избирательное активное внимание пользователя ИМК к соответствующей команде. Тем не менее волна Р300 и другие ЭЭГ-признаки наличия внимания к событию могут быть вызваны стимулами, привлекающими внимание человека неявным образом и не обладающими явным практическим интересом для человека. Наличие такого непроизвольного внимания может быть обусловлено субъективной значимостью тех или иных стимулов для человека ввиду его личного опыта, или психоэмоционального статуса [8, 9]. Таким образом, парадигма предъявления стимулов ИМК-РЗО0 подходит для оценки на основе ЭЭГ-реакций особенностей восприятия субъектом определенной информации и распознавания фокуса неявного интереса к некоторым классам внешних стимулов. При этом в такой системе стимулы могут предъявляться в так называемой парадигме пассивного внимания, когда от испытуемых не требуется реагировать на определенный класс стимулов, а нужно лишь смотреть на появляющиеся зрительные стимулы [10] Оправданность этого подхода объясняется тем, что даже в отсутствии осознанного восприятия выделяющегося биологически или эмоционально стимула он в любом случае может вызывать реакцию [11], поскольку значим для субъекта. Поэтому такой стимул можно рассматривать «целевым» в контексте ИМК, и к нему могут быть применимы существующие подходы классификации на основе анализа ЭЭГ.

Наиболее логичным представляется использование в подобных методиках эмоционально окрашенного контента на фоне нейтрального [12]. Подобные системы могут быть использованы для автоматического распознавания повышенного состояния возбуждения среди людей с профессиями высокой эмоциональной нагрузки [13], а также для инструментальной диагностики нарушения эмоционального восприятия, например, при аутизме [14, 15].

Не менее интересно использование методик на базе ИМК-P300 для оценки состояния больных с нарушениями пищевого поведения. В частности, анализ показателей ЭЭГ в ответ на предъявление таким пациентам набора зрительных стимулов, связанных с анорексией, может служить дополнительным критерием мониторинга успешности проводимого лечения. У пациентов с нервной анорексией ранее на ЭЭГ-уровне были выявлены нарушения в восприятии эмоционально окрашенных стимулов [16]. Кроме того, у таких больных известны особенности волны Р300 и других компонентов ПСС в заданиях на внимание, которые свидетельствуют о снижении некоторых функций мозга [17].

Несмотря на существование ряда работ по выявлению особенностей показателей ЭЭГ в ответ на предъявление зрительных стимулов в различных условиях, на сегодняшний день не было предложено прототипа такой системы, которая могла бы на основе сигнала ЭЭГ распознавать фокус интереса к определенным классам стимулов. Исходя из этого, целями настоящей работы были выявление особенностей ПСС в ответ на предъявление стимулов различной эмоциональной значимости больным нервной анорексией в парадигме пассивного внимания, а также проверка гипотезы о возможном детектировании фокуса внимания к субъективно значимым стимулам на основе технологии ИМК-Р300.

\section{ПАЦИЕНТЫ И МЕТОДЫ}

В исследовании принимали участие 12 девушек подросткового возраста (11-16 лет), проходивших лечение в ГБУЗ «НПЦ психического здоровья детей и подростков им. Г. Е. Сухаревой ДЗМ». Критерии включения пациентов в исследование: наличие диагноза F50.0 «Нервная анорексия»; период реабилитации. Критерии исключения: наличие тяжелой соматической патологии; высокая степень белково-энергетической недостаточности; пребывание на строгом постельном режиме. У участников регистрировали ЭЭГ во время того, как они смотрели на экран, на котором предъявляли наборы фотоизображений.

Изображения с угловыми размерами 12,9 × 9,60 предъявляли на сером фоне в режиме одбол-парадигмы: в центре экрана последовательно появлялись стимулы, в промежутках между ними экран оставался пустым. Длительность предъявления стимула составляла 200 мс, межстимульный интервал - 500 мс. В качестве значимых стимулов использовали два типа изображений: фотографии еды и частей тела людей с истощением. Нейтральными («незначимыми») стимулами служили изображения предметов, животных, геометрических фигур, 
пейзажей и др., взятые из базы IAPS [18], из которой также частично были отобраны изображения еды. Основная часть значимых стимулов была получена на основе анализа интернет-каналов анорексической тематики. Bce изображения предварительно оценивал врач, постоянно работавший с пациентами, участвовавшими в исследовании. Все изображения были скомбинированы в наборы по 6 штук, 5 изображений набора относились к категории «незначимые», а одно изображение к категории «значимые». Каждый набор стимулов предъявляли в рамках одной записи ЭЭГ длительностью менее минуты; записи были разделены короткими паузами. Предъявление осуществляли в виде стимульных последовательностей: одна такая последовательность включает появление каждого изображения по одному разу в случайном порядке. Одна запись ЭЭГ состояла из 10 стимульных последовательностей. Наборы изображений предъявляли каждому испытуемому в псевдослучайном порядке, при этом записи со стимульным материалом на основе изображений еды и частей тела чередовали. Общее количество записей для стимулов на основе изображений еды и частей тела составляло обычно 10 и 12 соответственно у каждого испытуемого.

ЭЭГ регистрировали монополярно в отведениях $\mathrm{Cz}$, P3, Pz, P4, PO7, PO8, O1, О2 с ресерентным электродом на мочке левого уха при помощи усилителя NVX52 с частотой дискретизации сигнала 500 Гц. Запись данных и управление предъявлением стимулов осуществляли в программной среде ВCl2000 (www.bci2000.org).

Выделение и анализ ПСС осуществляли в среде MATLAB 7.1 (MathWorks; CШA). Для этого сигнал ЭЭГ подвергали полосной фильтрации в диапазоне 0,5-20 Гц (фильтр Баттерворта), а затем разбивали на эпохи относительно момента подачи стимула с границами от 0,1 до 0,7 с. После удаления из общего массива эпох, содержащих глазодвигательные артефакты, происходило разделение эпох на целевые («значимые») и нецелевые («незначимые»). Эпохи разделяли также на два блока в зависимости от типа значимого изображения (еда или часть тела). Количество целевых и нецелевых эпох выравнивали за счет исключения части последних. Отдельно усредняли эпохи для целевых и нецелевых стимулов. Таким образом, целевые и нецелевые ПСС получали во всех каналах ЭЭГ в двух блоках для каждого испытуемого. Кроме того, выделяли «разностные» ПСС, получаемые вычитанием нецелевых кривых из целевых.

Амплитуды компонентов P300, N1 и LPP измеряли как максимальное либо минимальное значение в индивидуально подобранном для каждого участника временном окне. Амплитуды компонента Р300 были измерены в каналах Cz и Pz, амплитуды компонентов N1 и LPP — в каналах PO7, P08, О1 и О2. Не все компоненты проявлялись у каждого из испытуемых и во всех каналах, поэтому в описании результатов указывали число испытуемых (n), у которых тот или иной анализ производили.

Чтобы оценить эффективность распознавания по ЭЭГ наличия особой реакции внимания к значимому стимулу, был смоделирован подход классификации целевых команд в ИМК-Р300 на основе линейного дискриминанта Фишера. Обучение и тестирование классификатора происходило с использованием кросс-валидации по двум классам целевым и нецелевым стимулам. В ходе тестирования в каждой попытке (одна запись) в соответствии с обученным классисикатором определяли один наиболее выделяющийся из шести стимул. Если распознанное таким образом стимул-изображение принадлежало к категории «значимые», то попытку классификации считали успешной.

По результатам вычисления амплитуд ПСС и точности классификации проводили статистический анализ данных с помощью пакета STATISTICA 7.0 (StatSoft; CШA), и рассчитывали средние значения для каждой величины В обоих блоках. При нормальном распределении выборок использовали парный критерий Стьюдента, в случае отклонения распределения от нормального - ранговый критерий Уилкоксона.

\section{РЕЗУЛЬТАТЫ ИССЛЕДОВАНИЯ}

У большинства пациентов с нервной анорексией на индивидуальных разностных кривых были идентифицированы пики N1 (латентность 120-180 мс), P300 (350-450мс) и LPP (550-700 мс). Эти пики видны также на усредненных по всей группе целевых и нецелевых ПСС, а также разностных кривых (рис. 1). Соотношение амплитуд целевых и нецелевых ПСС, отображаемое на разностных кривых, позволяет судить о том, насколько сильно привлекали внимание значимые в контексте задания стимулы по сравнению с незначимыми. Поскольку индивидуальная латентность пиков варьировалась и не все пики можно наблюдать у всех испытуемых, на групповом усреднении компоненты ПСС в некоторых отведениях выглядят размытыми и мало выраженными, поэтому рис. 1 отражает лишь общую картину. Средние значения всех анализируемых компонентов в целевых, нецелевых и разностных кривых представлены в таблице.

В обоих блоках амплитуда компонента Р300 на целевых кривых была выше, чем на нецелевых: как в канале Cz ( $p<0,01 ; n=11$ и $p<0,01, n=12$, парный критерий Стьюдента), так и в канале $\mathrm{Pz}(p<0,01, n=12$ и $p<0,01$, $n=11)$ при предъявлении изображений пищи и частей тела людей с истощением соответственно (таблица). При сравнении амплитуд Р300 на разностных кривых между двумя типами стимулов не было выявлено значимых различий (рис. 2).

Амплитуды компонента N1 в целевых ПСС были выше, чем в нецелевых, как при предъявлении изображений пищи ( $p<0,05, n=9 ; p<0,05, n=10 ; p<0,05, n=9 ; p<0,05$, $n=11$, в отведениях РО7, РО8, О1, О2 соответственно, парный критерий Стьюдента), так и при предъявлении изображений частей тела ( $p<0,01, n=9 ; p<0,01, n=12$; $p<0,01, n=12 ; p<0,01, n=11$, критерий Уилкоксона). Несмотря на более высокие средние значения амплитуд N1 на разностных кривых для изображений частей тела по сравнению с изображениями пищи (рис. 1 и таблица), значимые различия между двумя типами стимулов выявлены только в отведении РО8 ( $p<0,05, n=10$; критерий Уилкоксона) (рис. 2).

Компонент LPP имел наибольшую амплитуду в затылочных отведениях. Амплитуды LPP в целевых ПСС были выше, чем в нецелевых, как при предъявлении изображений пищи $(p<0,01, n=8 ; p<0,01, n=9 ; p<0,05, n=8 ; p<0,01$, $n=9$, в отведениях РО7, РО8, О1, О2 соответственно, парный критерий Стьюдента), так и при предъявлении изображений частей тела ( $p<0,01, n=9 ; p<0,01, n=10$; $p<0,01, n=10 ; p<0,01, n=10)$. В среднем для изображений частей тела амплитуды LPP были выше, чем для изображений пищи, однако различия были значимы только в отведениях РО7 и РО8 $(p<0,05, n=7 ; p<0,05$, $n=8$ соответственно) (рис. 2). 
P3

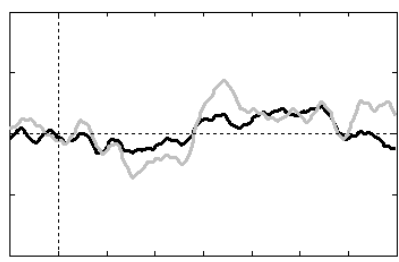

$\mathrm{PO} 7$

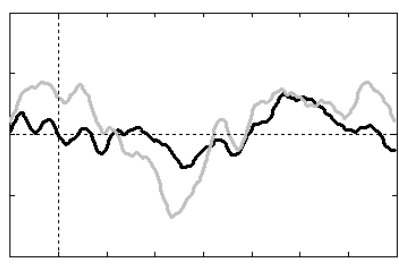

01

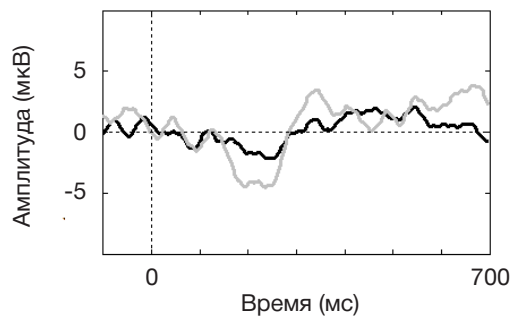

Cz

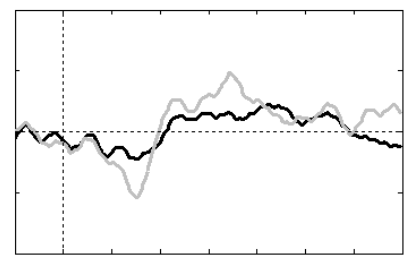

Pz

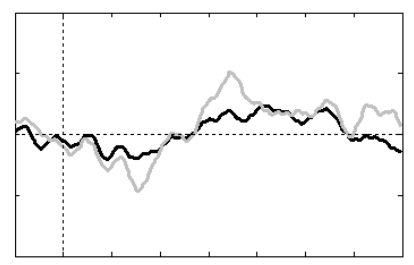

P4

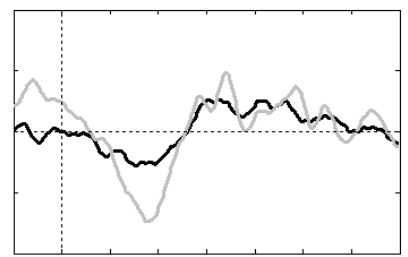

P08

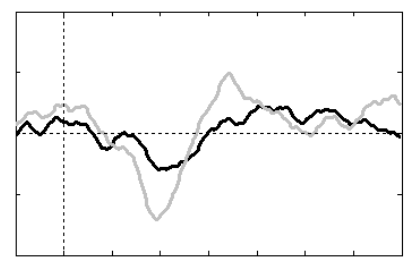

$\mathrm{O} 2$

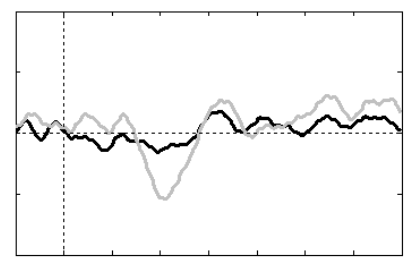

Рис. 1. Усредненные по группе разностные ПСС ( $n=10)$. Черная линия - стимулы-изображения пищи, серая линия - стимулы-изображения частей тела людей с истощением. По вертикали — амплитуда (мкВ), по горизонтали — время (мс). Вертикальная пунктирная линия (0 мс) соответствует времени подачи стимула. Над каждой кривой указано название отведения ЭЭГ

На рис. 3 представлены средние значения точности классификации значимых стимулов среди нейтральных в двух блоках. Средняя точность распознавания классификатором стимулов с изображениями пищи составила 59,1 \pm 5,3\% и была ниже, чем для стимулов с изображениями частей тела людей с истощением, для которых составила 89,1 $\pm 2,3 \%(p=0,0002, n=12$, парный критерий Стьюдента).

\section{ОБСУЖДЕНИЕ РЕЗУЛЬТАТОВ}

Главным результатом работы стал ряд выявленных особенностей ПСС при пассивном восприятии эмоционально значимых изображений, а также показанная возможность распознавания ЭЭГ-реакций внимания к таким стимулам среди нейтрального стимульного контента с точностью, близкой к современным ИМК-системам.

Известно, что для людей, относящихся к высокой группе риска развития расстройств пищевого поведения, характерна повышенная восприимчивость к эмоционально значимым стимулам, которые связаны с формой и массой тела [19]. Такую особую субъективную значимость подобных стимулов для больных с нервной анорексией можно объяснить тем, что в ее развитии важную роль играет особое отношение к своему телу с формированием синдрома дисморфофобии/дисморфомании на фоне

Таблица. Значения амплитуд компонентов Р300, N1 и LPP в ответ на предъявление стимулов с изображениями пищи и частей тела людей с истощением (приведено среднее \pm стандартная ошибка среднего, мкВ)

\begin{tabular}{|c|c|c|c|c|c|c|}
\hline & \multicolumn{3}{|c|}{ Изображения пищи } & \multicolumn{3}{|c|}{ Изображения частей тела } \\
\hline \multicolumn{7}{|c|}{ P300 } \\
\hline Отведение & Целевые & Нецелевые & Разностные & Целевые & Нецелевые & Разностные \\
\hline $\mathrm{Cz}$ & $4,5 \pm 1,1$ & $1,4 \pm 0,7$ & $5,8 \pm 0,9$ & $4,1 \pm 0,6$ & $0,6 \pm 0,4$ & $5,9 \pm 0,9$ \\
\hline $\mathrm{Pz}$ & $5,0 \pm 0,6$ & $2,1 \pm 0,6$ & $5,5 \pm 0,9$ & $5,7 \pm 0,8$ & $2,2 \pm 0,8$ & $6,0 \pm 0,8$ \\
\hline \multicolumn{7}{|c|}{ N1 } \\
\hline $\mathrm{PO} 7$ & $-6,1 \pm 1,6$ & $-3,0 \pm 0,9$ & $-3,9 \pm 1,0$ & $-4,5 \pm 1,8$ & $0,5 \pm 0,9$ & $-7,3 \pm 1,6$ \\
\hline PO8 & $-5,8 \pm 2,1$ & $-2,7 \pm 1,4$ & $-4,7 \pm 1,1$ & $-6,6 \pm 1,6$ & $-2,2 \pm 1,8$ & $-8,7 \pm 1,6$ \\
\hline 01 & $-4,6 \pm 2,2$ & $-1,7 \pm 1,6$ & $-4,5 \pm 1,1$ & $-4,1 \pm 1,8$ & $0,1 \pm 1,7$ & $-6,3 \pm 0,7$ \\
\hline $\mathrm{O} 2$ & $-4,8 \pm 2,3$ & $-2,4 \pm 1,8$ & $-4,0 \pm 0,8$ & $-4,3 \pm 1,9$ & $-0,3 \pm 2,2$ & $-6,4 \pm 0,6$ \\
\hline \multicolumn{7}{|c|}{ LPP } \\
\hline $\mathrm{PO} 7$ & $0,7 \pm 0,8$ & $-2,1 \pm 0,9$ & $4,2 \pm 0,6$ & $6,8 \pm 2,7$ & $-1,6 \pm 0,8$ & $10,9 \pm 2,4$ \\
\hline $\mathrm{PO} 8$ & $0,4 \pm 1,2$ & $-2,1 \pm 0,9$ & $4,1 \pm 0,8$ & $4,9 \pm 1,0$ & $-0,8 \pm 1,0$ & $8,0 \pm 1,3$ \\
\hline 01 & $-0,4 \pm 1,3$ & $-3,6 \pm 1,2$ & $4,4 \pm 0,9$ & $2,5 \pm 1,4$ & $-2,9 \pm 0,8$ & $8,4 \pm 1,4$ \\
\hline $\mathrm{O} 2$ & $-1,5 \pm 1,0$ & $-4,5 \pm 1,1$ & $4,5 \pm 0,8$ & $1,8 \pm 1,2$ & $-2,9 \pm 0,9$ & $8,5 \pm 1,4$ \\
\hline
\end{tabular}


низкого уровня самооценки и самоуважения [19, 20]. Нами были выявлены более высокие амплитуды компонентов ПСС в ответ на предъявление изображений пищи или частей тела людей с истощением, что говорит о субъективной значимости этих стимулов для пациентов, хотя сила реакции в ответ на два типа изображений различалась.

Исходя из литературных данных, более высокую амплитуду ПСС в ответ на значимые стимулы можно было бы объяснить их редкостью среди нейтральных стимулов: в одбол-парадигме редкие целевые стимулы вызывают более высокие ответы [21]. При этом, в отличие от классической одбол-парадигмы с простыми сильно различающимися двумя классами стимулов, все изображения в нашей работе были одинаково разнородными как внутри классов значимые/незначимые, так и между классами. Это говорит о том, что полученные в работе различия не связаны с графическими особенностями изображений, а вызваны исключительно субъективной значимостью смыслового содержания этой группы стимулов для пациентов. Так, предъявление изображений частей тела вызывало существенно более сильную реакцию в ПСС по сравнению с изображениями пищи, несмотря на одинаковую вероятность появления значимого стимула в обоих случаях. Это может говорить о том, что реакция на выделяющийся из контекста стимул обусловлена не просто его редкостью, но и его особой эмоциональной значимостью для пациентов. Такое предположение подтверждается и тем, что амплитуда волны Р300, которая отражает частотные характеристики стимула, не различалась для двух типов изображений, в то время как другие компоненты ПСС и точность классификации были чувствительны к типу стимула.

Одним из самых важных результатов описываемой работы можно считать крайне высокие значения точности классификации по ЭЭГ-реакциям субъективно значимых стимулов среди всех изображений. Точность для изображений пищи составила 59\%, а для изображений частей тела - 89\%. Следует отметить, что эти результать получены в задании без привлечения активного внимания к стимулам. Такая точность не просто многократно превышает случайный уровень распознавания в 16,7\% (выбор одного из шести стимулов), но близка к значениям, достигаемым в ИМК-Р300, где пользователь, напротив, активно фокусирует внимание на целевом стимулекоманде, игнорируя остальные стимулы [22, 23].

Описываемое в настоящей работе исследование является в большой мере пилотным, и его ценность определяется адаптацией существующих подходов ИМК для создания методик и систем детектирования субъективных фокусов внимания. Потому одним из ограничений работы является отсутствие контрольной группы здоровых испытуемых. При этом в качестве контроля можно рассматривать режим с предъявлением изображений пищи по сравнению с предъявлением изображений частей тела: компонентный состав ПСС различался между режимами, несмотря на одинаковые вероятностные характеристики значимого стимула и идентичный набор незначимых стимулов (см. выше). Полученные результаты можно также сравнить с результатами другой недавней работы авторского коллектива, где здоровые испытуемые выполняли похожее задание: пассивно наблюдали на экране различные изображения, часть из которых была эмоционально выделяющейся из контекста, но не несла для испытуемых субъективной значимости [12]. Амплитуды компонентов в ответ на выделяющиеся стимулы были значительно ниже, чем в настоящем исследовании, а точность классификации составляла 40-45\%. По некоторым данным, у больных нервной анорексией наблюдали сниженные ПСС-ответы на пищевые зрительные стимулы по сравнению со здоровыми испытуемыми [24]. В нашем же исследовании изображения частей тела людей с истощением вызывали существенно более высокую реакцию, подтвержденную на ЭЭГ, чем изображения пищи. Это может свидетельствовать о способностях первых привлекать внимание в большей степени и подчеркивает их особую значимость для больных нервной анорексией.

Компонент N1 отражает процессы фиксации на определенном стимуле, а также связан с эмоциональным восприятием [25], что позволяет судить о наличии внимания пациентов к изображениям пищи и частей тела, а также о том, что значимость данных стимулов была обусловлена эмоциональной реакцией на них. Компонент

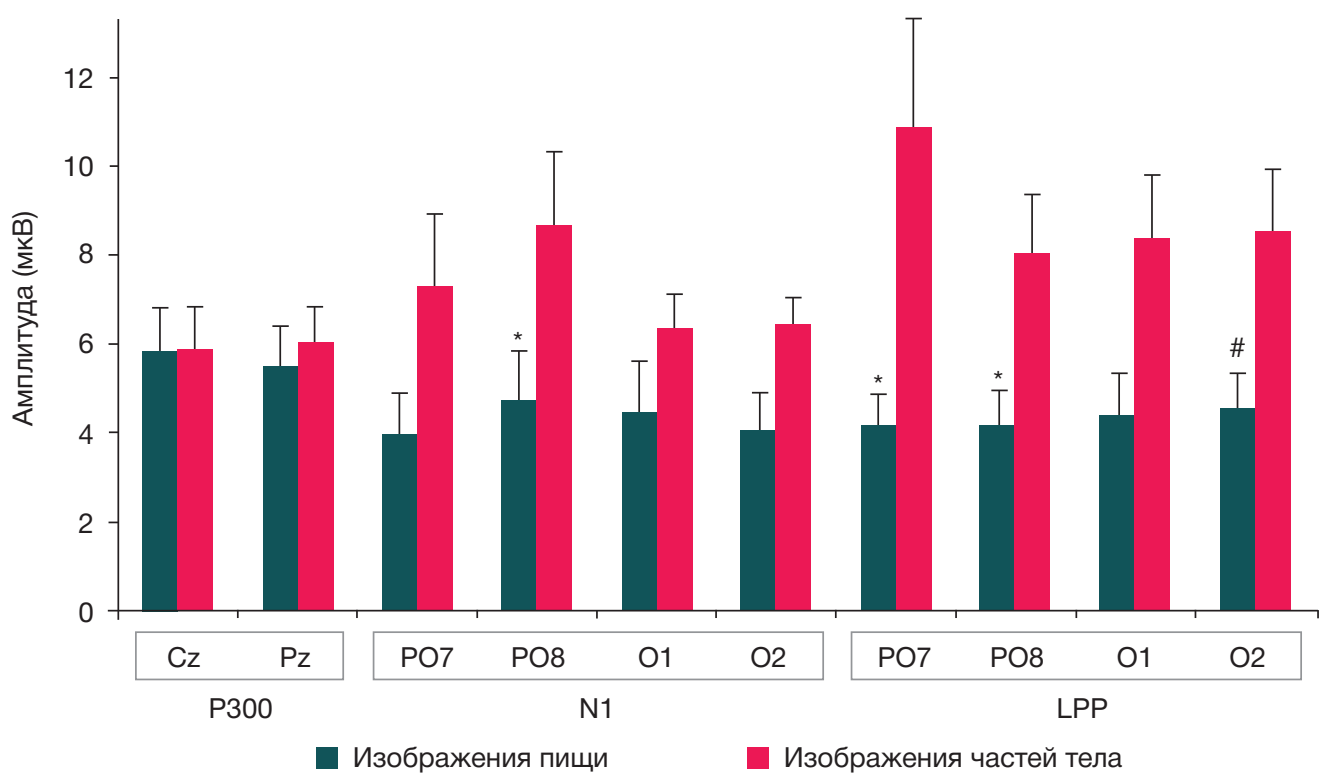

Рис. 2. Средняя по группе амплитуда компонентов P300, N1 и LPP для двух типов стимулов (изображения пищи и частей тела людей с истощением). Показаны среднее и стандартная ошибка среднего. Различия между блоками: * $-p<0,05, \#-p<0,1$ 
LPP связывают с поздними стадиями эмоционального процессинга зрительных стимулов [26]. Это также может служить подтверждением того, что особая ЭЭГ-реакция в ответ на используемые в исследовании значимые изображения определяется особенностями когнитивной обработки, а не просто частотностью их появления среди остальных стимулов. В целом более высокие амплитуды компонентов N1 и LPP, а также значения точности классификации для изображений частей тела людей с истощением по сравнению с изображениями пищи подтверждают предположение о том, что основной причиной анорексии может являться особое отношение к своему телу, искаженное восприятие его формы и размера, навязчивое желание соответствовать определенным физическим стандартам, а не собственно неприятие пищи [19].

\section{ВЫВОДЫ}

Использованный метод выявления непроизвольного внимания при предъявлении изображений пищи и частей тела истощенных людей обеспечил высокую точность классификации реакций непроизвольного внимания к эмоционально значимым стимулам у больных нервной анорексией. Развитие такой методики может способствовать созданию систем детектирования по ЭЭГ эмоциональных фокусов внимания. Подобные системы, в частности, могут быть использованы в диагностике состояния пациентов для определения особенностей их эмоционального восприятия на разных этапах лечения. Была выявлена более сильная реакция, подтвержденная на ЭЭГ, в ответ на предъявление изображений частей тела истощенных людей по сравнению с изображениями пищи. Это, с одной стороны, может подтверждать известные данные о факторах развития нервной анорексии, а, с другой стороны, открывает потенциал использования соответствующего стимульного материала в упомянутых системах.

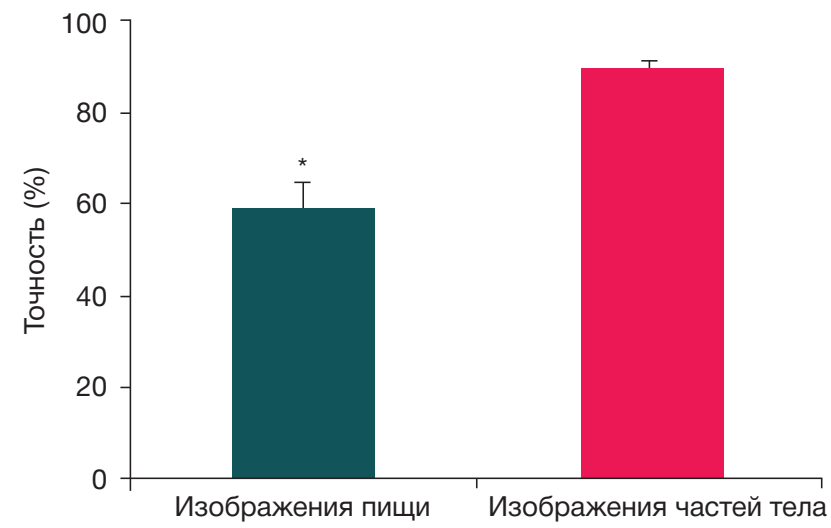

Рис. 3. Средняя по группе точность классификации с помощью алгоритма ИМК значимых стимулов среди нейтральных в двух блоках (изображения пищи и частей тела людей с истощением). Показаны среднее и стандартная ошибка среднего. Различия между блоками: * $-p<0,05$

\section{Литература}

1. McFarland DJ, Vaughan TM. $\mathrm{BCl}$ in practice. Progress in brain research. 2016; 228: 389404. Available from: https://doi org/10.1016/bs.pbr.2016.06.005.

2. Wolpaw JR. Brain-computer interfaces as new brain output pathways. J Physiol. 2007; 579 (3): 613-9. Available from: https:// doi.org/10.1113/jphysiol.2006.125948.

3. Alqasemi R, Dubey R. A 9-DoF Wheelchair-Mounted Robotic Arm System: Design, Control, Brain-Computer Interfacing, and Testing. Journal of Advances in Robot Manipulators In Tech. 2010: 51-78. Available from: https://doi.org/10.5772/9678.

4. Lopes AC, Pires G, Vaz L, Nunes U. Wheelchair navigation assisted by human-machine shared-control and a P300-based brain computer interface. International Conference on Intelligent Robots and Systems (IROS). 2011: 2438-44. Available from: https://doi.org/10.1109/iros.2011.6094748.

5. Vidaurre C, Klauer C, Schauer T, Ramos-Murguialday A, Müller KR EEG-based $\mathrm{BCl}$ for the linear control of an upper-limb neuroprosthesis. Medical Engineering \& Physics. 2016; 38 (11): 1195-204. Available from: https://doi.org/10.1016/j.medengphy.2016.06.010.

6. Rezeika A, Benda M, Stawicki P, Gembler F, Saboor A, Volosyak I. Brain-Computer Interface Spellers: A Review. Brain Sciences. 2018; 8 (4): 57. Available from: https://doi.org/10.3390/brainsci8040057.

7. Farwell LA, Donchin E. Talking off the top of your head: toward a mental prosthesis utilizing event-related brain potentials. Electroencephalography and Clinical Neurophysiology. 1988; 70: 510-23. Available from: https://doi.org/10.1016/0013-4694(88)90149-6.

8. Asmaro D, Jaspers-Fayer F, Sramko V, Taake I, Carolan P, Liotti M Spatiotemporal dynamics of the hedonic processing of chocolate images in individuals with and without trait chocolate craving Appetite. 2012; 58 (3): 790-9. Available from: https://doi. org/10.1016/j.appet.2012.01.030.

9. Asmaro D, Carolan PL, Liotti M. Electrophysiological evidence of early attentional bias to drug-related pictures in chronic cannabis users. Addict Behav. 2014; 39 (1): 114-21. Available from: https:// doi.org/10.1016/j.addbeh.2013.09.012

10. Olofsson, JK, Nordin S, Sequeira H, Polich J. Affective picture

processing: an integrative review of ERP findings. Biological psychology. 2008; 77 (3): 247-65. Available from: https://doi. org/10.1016/j.biopsycho.2007.11.006.

11. Ohman A, Flykt A, Esteves F. Emotion drives attention: detecting the snake in the grass. Journal of experimental psychology: general. 2001; 130 (3): 466-78. Available from: https://doi. org/10.1037//0096-3445.130.3.466.

12. Ганин И. П., Косиченко Е. А., Каплан А. Я. Особенности электроэнцефалографических реакций на эмоционально значимые стимулы в технологии интерфейса мозг-компьютер на волне Р300. Журн. высш. нерв. деят. 2017; 67 (4): 453-63. Available from: https://doi.org/10.7868/s0044467717040074.

13. Singh $M$, Singh $M$, Goyal M. Selection of attribute combinations of ERP's for classification of emotions along arousal axis. International Journal of Information Technology \& Knowledge Management. 2015; 8 (2): 142-9. Available from: https://doi. org/10.141079/IJITKM.2015.801.

14. Liu TL, Wang PW, Yang YC, Hsiao RC, Su YY, Shyi GC, Yen CF. Deficits in facial emotion recognition and implicit attitudes toward emotion among adolescents with high functioning autism spectrum disorder. Compr Psychiatry. 2019; (90): 7-13. Available from: https://doi.org/10.1016/j.comppsych.2018.12.010.

15. Stavropoulos KK, Viktorinova M, Naples A, Foss-Feig J, McPartland JC. Autistic traits modulate conscious and nonconscious face perception. Soc Neurosci. 2016; (10): 1-12. Available from: https:// doi.org/10.1080/17470919.2016.1248788.

16. Hatch A, Madden S, Kohn MR, Clarke S, Touyz S, Gordon E et al. Emotion brain alterations in anorexia nervosa: a candidate biological marker and implications for treatment. Journal of psychiatry \& neuroscience: JPN. 2010; 35 (4): 267-74. Available from: https://doi.org/10.1503/jpn.090073.

17. Bradley SJ, Taylor MJ, Rovet JF, Goldberg E, Hood J, Wachsmuth R, et al. Assessment of brain function in adolescent anorexia nervosa before and after weight gain. Journal of Clinical and Experimental Neuropsychology. 1997; 19 (1): 20-33. Available from: https:// doi.org/10.1080/01688639708403833. 
18. Lang PJ, Bradley MM, Cuthbert BN. International affective picture system (IAPS): Affective ratings of pictures and instruction manual. Technical Report A-8. University of Florida, Gainesville, FL. 2008.

19. Meijboom A, Jansen A, Kampman M, Schouten E. An experimental test of the relationship between self-esteem and concern about body shape and weight in restrained eaters. International Journal of Eating Disorders. 1999; 25 (3): 327-34. Available from: https://doi.org/10.1002/(sici)1098-108x(199904)25:3<327::aideat11>3.0.co;2-5.

20. Blechert J, Ansorge U, Beckmann S, \& Tuschen-Caffier B. The undue influence of shape and weight on self-evaluation in anorexia nervosa, bulimia nervosa and restrained eaters: a combined ERP and behavioral study. Psychological Medicine. 2011; 41 (1): 185-94. Available from: https://doi.org/10.1017/s0033291710000395.

21. Squires NK, Squires KC, Hillyard SA. Two Varieties of Long-latency positive Waves Evoked by Unpredictavle Audiatory Stimuli in Man. Electroencephalography and Clinical Neurophysiology. 1975; (38): 387-401. Available from: https://doi.org/10.1016/00134694(75)90263-1.

22. Guger C, Daban S, Sellers E, Holzner C, Krausz G, Carabalona R, et al. How many people are able to control a P300-based brain- computer interface (BCl)? Neurosci Lett. 2009; 462 (1): 94-8. Available from: https://doi.org/10.1016/j.neulet.2009.06.045.

23. Ганин И. П., Каплан А. Я. Интерфейс мозг-компьютер на основе волны Р300: предъявление комплексных стимулов «подсветка + движение». Журнал высшей нервной деятельности. 2014; 64 (1): 32-40. Доступно по ссылке: https://doi.org/10.7868/s0044467714010067.

24. Nikendei C, Friederich HC, Weisbrod M, Walther S, Sharma A Herzog W, et al. Event-related potentials during recognition of semantic and pictorial food stimuli in patients with anorexia nervosa and healthy controls with varying internal states of hunger. Psychosomatic medicine. 2012; 74 (2): 136-145. Available from: https://doi.org/10.1097/psy.0b013e318242496a.

25. Sprengelmeyer R, Jentzsch I. Event related potentials and the perception of intensity in facial expressions. Neuropsychologia. 2006; (44): 2899-06. Available from: https://doi.org/10.1016/j. neuropsychologia.2006.06.020.

26. Foti D, Hajcak G, Dien J. Differentiating neural responses to emotional pictures: Evidence from temporal-spatial PCA. Psychophysiology. 2009; 46 (3): 521-30. Available from: https:// doi.org/10.1111/j.1469-8986.2009.00796.x.

\section{References}

1. McFarland DJ, Vaughan TM. BCl in practice. Progress in brain research. 2016; 228: 389404. Available from: https://doi. org/10.1016/bs.pbr.2016.06.005.

2. Wolpaw JR. Brain-computer interfaces as new brain output pathways. J Physiol. 2007; 579 (3): 613-9. Available from: https:// doi.org/10.1113/jphysiol.2006.125948.

3. Alqasemi R, Dubey R. A 9-DoF Wheelchair-Mounted Robotic Arm System: Design, Control, Brain-Computer Interfacing, and Testing. Journal of Advances in Robot Manipulators In Tech. 2010: 51-78. Available from: https://doi.org/10.5772/9678.

4. Lopes AC, Pires G, Vaz L, Nunes U. Wheelchair navigation assisted by human-machine shared-control and a P300-based brain computer interface. International Conference on Intelligent Robots and Systems (IROS). 2011: 2438-44. Available from: https://doi.org/10.1109/iros.2011.6094748.

5. Vidaurre C, Klauer C, Schauer T, Ramos-Murquialday A, Müller KR. EEG-based $\mathrm{BCl}$ for the linear control of an upper-limb neuroprosthesis. Medical Engineering \& Physics. 2016; 38 (11): 1195-204. Available from: https://doi.org/10.1016/j.medengphy.2016.06.010.

6. Rezeika A, Benda M, Stawicki P, Gembler F, Saboor A, Volosyak I. Brain-Computer Interface Spellers: A Review. Brain Sciences. 2018; 8 (4): 57. Available from: https://doi.org/10.3390/brainsci8040057.

7. Farwell LA, Donchin E. Talking off the top of your head: toward a mental prosthesis utilizing event-related brain potentials. Electroencephalography and Clinical Neurophysiology. 1988; 70: 510-23. Available from: https://doi.org/10.1016/0013-4694(88)90149-6.

8. Asmaro D, Jaspers-Fayer F, Sramko V, Taake I, Carolan P, Liotti M. Spatiotemporal dynamics of the hedonic processing of chocolate images in individuals with and without trait chocolate craving. Appetite. 2012; 58 (3): 790-9. Available from: https://doi. org/10.1016/j.appet.2012.01.030.

9. Asmaro D, Carolan PL, Liotti M. Electrophysiological evidence of early attentional bias to drug-related pictures in chronic cannabis users. Addict Behav. 2014; 39 (1): 114-21. Available from: https:// doi.org/10.1016/i.addbeh.2013.09.012.

10. Olofsson, JK, Nordin S, Sequeira H, Polich J. Affective picture processing: an integrative review of ERP findings. Biological psychology. 2008; 77 (3): 247-65. Available from: https://doi. org/10.1016/j.biopsycho.2007.11.006.

11. Ohman A, Flykt A, Esteves F. Emotion drives attention: detecting the snake in the grass. Journal of experimental psychology: general. 2001; 130 (3): 466-78. Available from: https://doi. org/10.1037//0096-3445.130.3.466.

12. Ganin IP, Kosichenko EA, Kaplan AYa. Osobennost jelektrojencefalograficheskih reakcij na jemocional'no znachimye stimuly v tehnologii interfejsa mozg-komp'juter na volne P300.
Zhurn vyssh nerv dejat. 2017; 67 (4): 453-63. Available from: https://doi.org/10.7868/s0044467717040074.

13. Singh $M$, Singh $M$, Goyal $M$. Selection of attribute combinations of ERP's for classification of emotions along arousal axis. International Journal of Information Technology \& Knowledge Management. 2015; 8 (2): 142-9. Available from: https://doi. org/10.141079/IJITKM.2015.801.

14. Liu TL, Wang PW, Yang YC, Hsiao RC, Su YY, Shyi GC, Yen CF. Deficits in facial emotion recognition and implicit attitudes toward emotion among adolescents with high functioning autism spectrum disorder. Compr Psychiatry. 2019; (90): 7-13. Available from: https://doi.org/10.1016/j.comppsych.2018.12.010.

15. Stavropoulos KK, Viktorinova M, Naples A, Foss-Feig J, McPartland JC. Autistic traits modulate conscious and nonconscious face perception. Soc Neurosci. 2016; (10): 1-12. Available from: https://doi.org/10.1080/17470919.2016.1248788.

16. Hatch A, Madden S, Kohn MR, Clarke S, Touyz S, Gordon E et al. Emotion brain alterations in anorexia nervosa: a candidate biological marker and implications for treatment. Journal of psychiatry \& neuroscience: JPN. 2010; 35 (4): 267-74. Available from: https://doi.org/10.1503/jpn.090073.

17. Bradley SJ, Taylor MJ, Rovet JF, Goldberg E, Hood J, Wachsmuth R, et al. Assessment of brain function in adolescent anorexia nervosa before and after weight gain. Journal of Clinical and Experimental Neuropsychology. 1997; 19 (1): 20-33. Available from: https:// doi.org/10.1080/01688639708403833.

18. Lang PJ, Bradley MM, Cuthbert BN. International affective picture system (IAPS): Affective ratings of pictures and instruction manual. Technical Report A-8. University of Florida, Gainesville, FL. 2008.

19. Meijboom A, Jansen A, Kampman M, Schouten E. An experimental test of the relationship between self-esteem and concern about body shape and weight in restrained eaters. International Journal of Eating Disorders. 1999; 25 (3): 327-34. Available from: https://doi.org/10.1002/(sici)1098-108x(199904)25:3<327::aideat $11>3.0 . c 0 ; 2-5$.

20. Blechert J, Ansorge U, Beckmann S, \& Tuschen-Caffier B. The undue influence of shape and weight on self-evaluation in anorexia nervosa, bulimia nervosa and restrained eaters: a combined ERP and behavioral study. Psychological Medicine. 2011; 41 (1): 185-94. Available from: https://doi.org/10.1017/s0033291710000395.

21. Squires NK, Squires KC, Hillyard SA. Two Varieties of Long-latency positive Waves Evoked by Unpredictavle Audiatory Stimuli in Man. Electroencephalography and Clinical Neurophysiology. 1975; (38): 387-401. Available from: https://doi.org/10.1016/00134694(75)90263-1

22. Guger C, Daban S, Sellers E, Holzner C, Krausz G, Carabalona R, 
et al. How many people are able to control a P300-based braincomputer interface (BCl)? Neurosci Lett. 2009; 462 (1): 94-8. Available from: https://doi.org/10.1016/j.neulet.2009.06.045.

23. Ganin IP, Kaplan AYa. Interfejs mozg-komp'juter na osnove volny P300: pred"javlenie kompleksnyh stimulov «podsvetka + dvizhenie». Zhurnal vysshej nervnoj dejatel'nosti. 2014; 64 (1): 32-40. Dostupno po ssylke: https://doi.org/10.7868/s0044467714010067.

24. Nikendei C, Friederich HC, Weisbrod M, Walther S, Sharma A, Herzog W, et al. Event-related potentials during recognition of semantic and pictorial food stimuli in patients with anorexia nervosa and healthy controls with varying internal states of hunger.
Psychosomatic medicine. 2012; 74 (2): 136-145. Available from: https://doi.org/10.1097/psy.0b013e318242496a.

25. Sprengelmeyer R, Jentzsch I. Event related potentials and the perception of intensity in facial expressions. Neuropsychologia 2006; (44): 2899-06. Available from: https://doi.org/10.1016/j. neuropsychologia.2006.06.020.

26. Foti D, Hajcak G, Dien J. Differentiating neural responses to emotional pictures: Evidence from temporal-spatial PCA. Psychophysiology. 2009; 46 (3): 521-30. Available from: https:// doi.org/10.1111/j.1469-8986.2009.00796.x. 